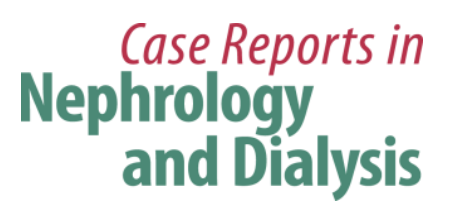

Case Rep Nephrol Dial 2016;6:143-148

\title{
Pregnant Woman with Atypical Hemolytic Uremic Syndrome Delivered a Healthy Newborn under Eculizumab Treatment
}

\author{
Erol Demir $^{\mathrm{a}}$ Halil Yazici $^{\mathrm{a}}$ Yasemin Ozluk $^{\mathrm{b}}$ Isin Kilicaslan ${ }^{\mathrm{b}}$ \\ Aydin Turkmen ${ }^{a}$ \\ aDivision of Nephrology, Department of Internal Medicine, Istanbul Faculty of Medicine,

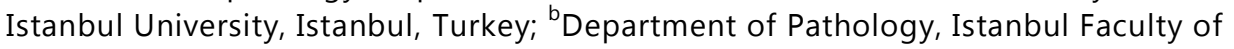 \\ Medicine, Istanbul University, Istanbul, Turkey
}

\section{Keywords}

Pregnancy-associated thrombotic microangiopathy · Pregnancy-associated atypical hemolytic uremic syndrome · Eculizumab

\begin{abstract}
Pregnancy-associated thrombotic microangiopathy is a very rare condition; however, it significantly increases fetal or maternal morbidity and mortality. Pregnancy may trigger atypical hemolytic uremic syndrome (aHUS) or thrombotic thrombocytopenic purpura. The risk for pregnancy-associated aHUS is highest during the second pregnancy. The outcome is usually poor with $50-60 \%$ mortality; renal dysfunction and hypertension are the rule in those who survive the acute episode. After the development of complement regulation mechanisms and aHUS pathogenesis, eculizumab has been widely used as a first-line treatment in aHUS. Eculizumab has been produced to minimize immunogenicity and Fc-mediated functions, including recruitment of inflammatory cells and complement activation, and using eculizumab in first-line treatment improves kidney function. Recent studies showed that early diagnosis and
\end{abstract}




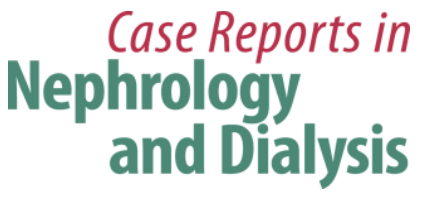

Case Rep Nephrol Dial 2016;6:143-148 DOI: $10.1159 / 000454946$

C 2016 The Author(s). Published by S. Karger AG, Basel www.karger.com/cnd

Demir et al.: Pregnant Woman with Atypical Hemolytic Uremic Syndrome Delivered a Healthy Newborn under Eculizumab Treatment

rapid use of eculizumab in first-line treatment improve outcomes. We demonstrate a case with pregnancy-triggered aHUS occurring in the second trimester, who was successfully treated and delivered a healthy baby under eculizumab treatment.

(C) 2016 The Author(s)

Published by S. Karger AG, Basel

\section{Introduction}

Pregnancy-associated thrombotic microangiopathy (TMA) is a very rare condition; however, it significantly increases fetal or maternal morbidity and mortality. Two different mechanisms may cause TMA in pregnancy. The first one is an acquired or genetic deficiency in ADAMTS13, a von Willebrand factor-processing enzyme. This leads to TMA with predominantly neurologic involvement and thrombocytopenia - thrombotic thrombocytopenic purpura. The other mechanism is mutations in one or more genes coding for proteins involved in the control of the alternative pathway of the complement system - atypical hemolytic uremic syndrome (aHUS). This type of TMA has predominantly renal involvement. Pregnancy may trigger aHUS or thrombotic thrombocytopenic purpura. More recently, complement dysregulation was also associated with HELLP (Hemolysis, Elevated Liver enzymes, and Low Platelet count) syndrome, which shares several features with pregnancy-associated TMA. HELLP syndrome is a variant or complication of preeclampsia and usually occurs during the third trimester of pregnancy $[1,2]$.

Eculizumab (Soliris) is an approved treatment for aHUS. It is a humanized monoclonal antibody that binds with high affinity to the human $\mathrm{C} 5$ complement protein and blocks the formation of proinflammatory $\mathrm{C} 5 \mathrm{a}$ and lytic $\mathrm{C} 5 \mathrm{~b}$ [3]. In this case report, we present a pregnant woman with aHUS who was successfully treated with eculizumab and delivered a healthy newborn.

\section{Case Report}

A previously healthy 17-year-old, 17-week-pregnant woman presented with a history of 1 week of headache and swelling in her legs. Mild pretibial edema was accompanied by abnormal urinalysis results (proteinuria $10 \mathrm{~g}$ /day).

Physical examination was unremarkable. Her blood pressure was 120/80 mm $\mathrm{Hg}$ and her gynecologic examination revealed normal fetus development. Laboratory workup revealed her blood creatinine level as $2.85 \mathrm{mg} / \mathrm{dL}$, hemoglobin $7.7 \mathrm{~g} / \mathrm{dL}$, platelets $52,000 / \mu \mathrm{L}$, albumin $3 \mathrm{~g} / \mathrm{dL}$, LDH $714 \mathrm{U} / \mathrm{L}$, haptoglobulin $7 \mathrm{mg} / \mathrm{dL}$, reticulocytes $8 \%$, direct and indirect Coombs tests were negative, and normal blood C3 and C4 levels. On peripheral blood smear, 8-10 schistocytes per field were found. ADAMTS13 activity was $80 \%$. We suspected aHUS and eculizumab was started after five sessions of plasmapheresis, due to unresponsiveness to plasmapheresis and persistent thrombocytopenia.

A biopsy was performed after improvement of platelets after 1 month of eculizumab treatment, which showed 25 glomeruli with endothelial swelling and mesangiolysis. Segmental duplication of glomerular basement membranes was also seen. Thrombi were present within arteriolar and glomerular capillary lumina. Immunofluorescence microscopy did not show any immune complex deposits (Fig. 1).

Under eculizumab treatment, her blood creatinine level decreased to $0.80 \mathrm{mg} / \mathrm{dL}$ and hemoglobin and platelets improved to $10 \mathrm{~g} / \mathrm{dL}$ and 202,000/ $\mu \mathrm{L}$, respectively. Albumin re- 


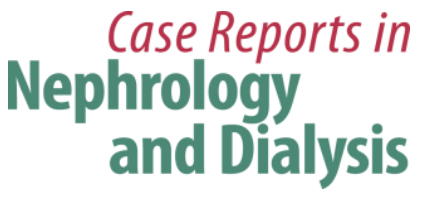

Case Rep Nephrol Dial 2016;6:143-148

DOI: $10.1159 / 000454946$

(c) 2016 The Author(s). Published by S. Karger AG, Basel www.karger.com/cnd

Demir et al.: Pregnant Woman with Atypical Hemolytic Uremic Syndrome Delivered a Healthy Newborn under Eculizumab Treatment

mained at $3 \mathrm{~g} / \mathrm{dL}$ and $\mathrm{LDH}$ decreased to $154 \mathrm{U} / \mathrm{L}$. She was treated with eculizumab every 15 days during pregnancy. Genotyping of patient was performed with Sanger sequencing of $\mathrm{CFH}$ and CFI genes. We identified two homozygosities for single-nucleotide polymorphism (SNPs) in complement factor $\mathrm{H}$ (exon 2 V62I and exon $9 \mathrm{H} 402 \mathrm{Y}$ ).

Her pregnancy was uneventful until at 31 weeks, at routine antenatal care, fetal distress was diagnosed by the gynecologist and she delivered a male baby at 31 weeks by caesarean section. The newborn was admitted to the neonatal intensive care unit due to respiratory distress, but he was discharged within 2 weeks with complete clinical recovery. The patient has been followed in our outpatient clinic with continued eculizumab treatment.

\section{Discussion}

Pregnancy may trigger aHUS. There are two forms of pregnancy-associated aHUS. One of them may occasionally develop as a complication of preeclampsia. Risk for pregnancyassociated aHUS is highest during the second pregnancy. Generally, it is expected that complete remission after delivery in this form occurs. On the other hand, the other form is usually manifested after delivery. The outcome is usually poor with $50-60 \%$ mortality; renal dysfunction and hypertension are the rule in those who survive the acute episode [4-6]. Our patient presented in the first trimester and she had renal dysfunction and nephrotic-range proteinuria. We classified our patient in the first group, but we did not make the diagnosis of preeclampsia because of the very early symptoms.

Eculizumab is a fully humanized hybrid IgG2/IgG4 monoclonal antibody, directed against human complement component $\mathrm{C} 5$. The drug has been produced to minimize immunogenicity and Fc-mediated functions, including recruitment of inflammatory cells and complement activation. A decade ago, plasma therapy was widely used as first-line, goldstandard treatment in aHUS. After the development of complement regulation mechanisms and aHUS pathogenesis, eculizumab has been widely used as a first-line treatment in aHUS. Recent studies showed that early diagnosis and rapid use of eculizumab in first-line treatment improve outcomes [7-9]. We diagnosed aHUS due to TMA, acute kidney injury, and thrombocytopenia with normal ADAMTS level and thrombi in arteriolar and glomerular capillary lumina in 3 days; however, we could not use eculizumab in first-line treatment because of our government health insurance restrictions due to unlabelled usage of eculizumab in aHUS treatment.

All patients with aHUS should be vaccinated against Neisseria meningitidis, Streptococcus pneumoniae, and Haemophilus influenzae type B infections. Although using eculizumab in pregnant women with paroxysmal nocturnal hemoglobinuria showed successful treatment outcomes with safety profile, there were only few case reports in pregnancy with aHUS [10, $11]$. We vaccinated our patient against $S$. pneumoniae, $H$. influenzae type $\mathrm{B}$, and $N$. meningitidis.

Glomerular thrombosis, intracapillary foamy cells, endocapillary swelling and hypercellularity, mesangiolysis, and double basement membranes are observed in aHUS kidney biopsy. Arterioles have thrombosis, endothelial swelling, or fibrinoid necrosis. Arteries have intimal swelling with various amounts of hypercellularity and thrombosis. aHUS diagnosis depends on clinical features and laboratory results; usually kidney biopsy is impossible due to bleeding (uremia, thrombocytopenia) diathesis [12]. However, we did kidney biopsy as soon as possible in order to eliminate other diagnoses, and then the rest of the pregnancy continued without any fetal and maternal problems until the 31st week of pregnancy. Preg- 
nancy-associated aHUS is associated with high maternal mortality and severe long-term morbidity and more than half of all patients develop end-stage renal disease in less than 1 month, most cases (79\%) developing in the postpartum period [12-14].

Six months after delivery, the patient remains well with normal renal function and no signs of TMA. We are continuing eculizumab due to ongoing risk of relapsing aHUS. There are only few reports of pregnancy-associated aHUS treated with eculizumab in the literature and treatment duration varied. Factor $\mathrm{H}$ mutation is also associated with high risk of relapse and poor prognosis [13-16]. We performed complement testing on our patient and identified polymorphic variants which are associated with aHUS and have been described previously.

Clinical and laboratory findings, kidney biopsy, and complement mutation must be done together for a definitive diagnosis of pregnancy-associated aHUS because it may be easily confused with HELLP syndrome or eclampsia. Also, it has been previously reported that fetal and maternal mortality rates increase due to incorrect diagnosis or delays in treatment [8]. Some case reports previously showed that pregnancy-associated aHUS usually occurs in the third trimester or postpartum $[14,15]$. This is the first case diagnosed during pregnancy and delivering successfully with eculizumab treatment in the literature.

In summary, we demonstrate a case with pregnancy-triggered aHUS occurring in the second trimester, who was successfully treated and delivered a healthy baby under eculizumab treatment.

\section{Acknowledgements}

Support for publishing fee was provided by Alexion.

\section{Statement of Ethics}

We obtained informed consent from the patient.

\section{Disclosure Statement}

The authors have declared no conflicts of interest.

\section{References}

-1 Fakhouri F, Jablonski M, Lepercq J, Factor H: Membrane cofactor protein, and factor I mutations in patients with hemolysis, elevated liver enzymes, and low platelet count syndrome. Blood 2008;112:4542-4545.

2 Fakhouri F, Roumenina L, Provot F, et al: Pregnancy-associated hemolytic uremic syndrome revisited in the era of complement gene mutations. J Am Soc Nephrol 2010;21:859-867.

3 Legendre CM, Licht C, Muus P, et al: Terminal complement inhibitor eculizumab in atypical hemolyticuremic syndrome. N Engl J Med 2013;368:2169-2181.

4 Nürnberger J, Philipp T, Witzke 0: Eculizumab for atypical hemolytic-uremic syndrome. N Engl J Med 2009;360:542-544.

-5 Zuber J, Fakhouri F, Roumenina LT: Use of eculizumab for atypical haemolytic uraemic syndrome and C3 glomerulopathies. Nat Rev Nephrol 2012;8:643-657. 


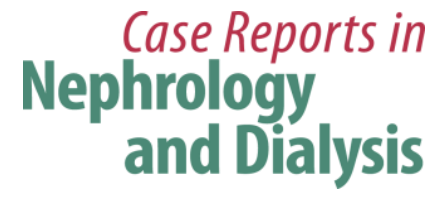

Case Rep Nephrol Dial 2016;6:143-148

(c) 2016 The Author(s). Published by S. Karger AG, Basel www.karger.com/cnd

Demir et al.: Pregnant Woman with Atypical Hemolytic Uremic Syndrome Delivered Healthy Newborn under Eculizumab Treatment

6 Kelly R, Arnold L, Richards S, et al: The management of pregnancy in paroxysmal nocturnal haemoglobinuria on long term eculizumab. Br J Haematol 2010;149:446-450.

7 Kelly RJ, Höchsmann B, Szer J, et al: Eculizumab in pregnant patients with paroxysmal nocturnal hemoglobinuria. N Engl J Med 2015;373:1032-1039.

8 Mandala EM, Gkiouzepas S, Kasimatis E, et al: Pregnancy-associated atypical hemolytic uremic syndrome (aHUS), treated with eculizumab. Blood 2014;124:5019.

-9 Amorim EDS, Blasco M, Quintana L, et al: Eculizumab in pregnancy-associated atypical hemolytic uremic syndrome: insights for optimizing management. J Nephrol 2015;28:641-645.

$\$ 10$ Delmas Y, Bordes C, Loirat C, et al: Post-partum atypical haemolytic-uraemic syndrome treated with eculizumab: terminal complement activity assessment in clinical practice. Clin Kidney J 2013;6:243244.

$\checkmark 11$ Canigral C, Moscardó F, Castro C, et al: Eculizumab for the treatment of pregnancy-related atypical hemolytic uremic syndrome. Ann Hematol 2014;93:1421-1422.

12 Ardissino G, Wally OM, Maria BG, et al: Eculizumab for atypical hemolytic uremic syndrome in pregnancy. Obstet Gynecol 2013;122:487-489.

13 Zschiedrich S, Prager EP, Kuehn EW, et al: Successful treatment of the postpartum atypical hemolytic uremic syndrome with eculizumab. Ann Intern Med 2013;2:76.

14 Kourouklaris A, Ioannou K, Athanasiou I, et al: Postpartum thrombotic microangiopathy revealed as atypical hemolytic uremic syndrome successfully treated with eculizumab: a case report. J Med Case Rep 2014;8:307.

15 Saad AF, Roman J, Wyble A, et al: Pregnancy-associated atypical hemolytic-uremic syndrome. AJP Rep 2016;6:125-128.

16 Hakobyan S, Tortajada A, Harris CL, et al: Variant-specific quantification of factor $\mathrm{H}$ in plasma reveals null alleles associated with atypical hemolytic uremic syndrome. Kidney Int 2010;78:782-788. 


\section{Case Reports in Nephrology and Dialysis}

Case Rep Nephrol Dial 2016;6:143-148 DOI: $10.1159 / 000454946$

(c) 2016 The Author(s). Published by S. Karger AG, Basel www.karger.com/cnd

Demir et al: Pregnant Woman with Atypical Hemolytic Uremic Syndrome Delivered a Healthy Newborn under Eculizumab Treatment

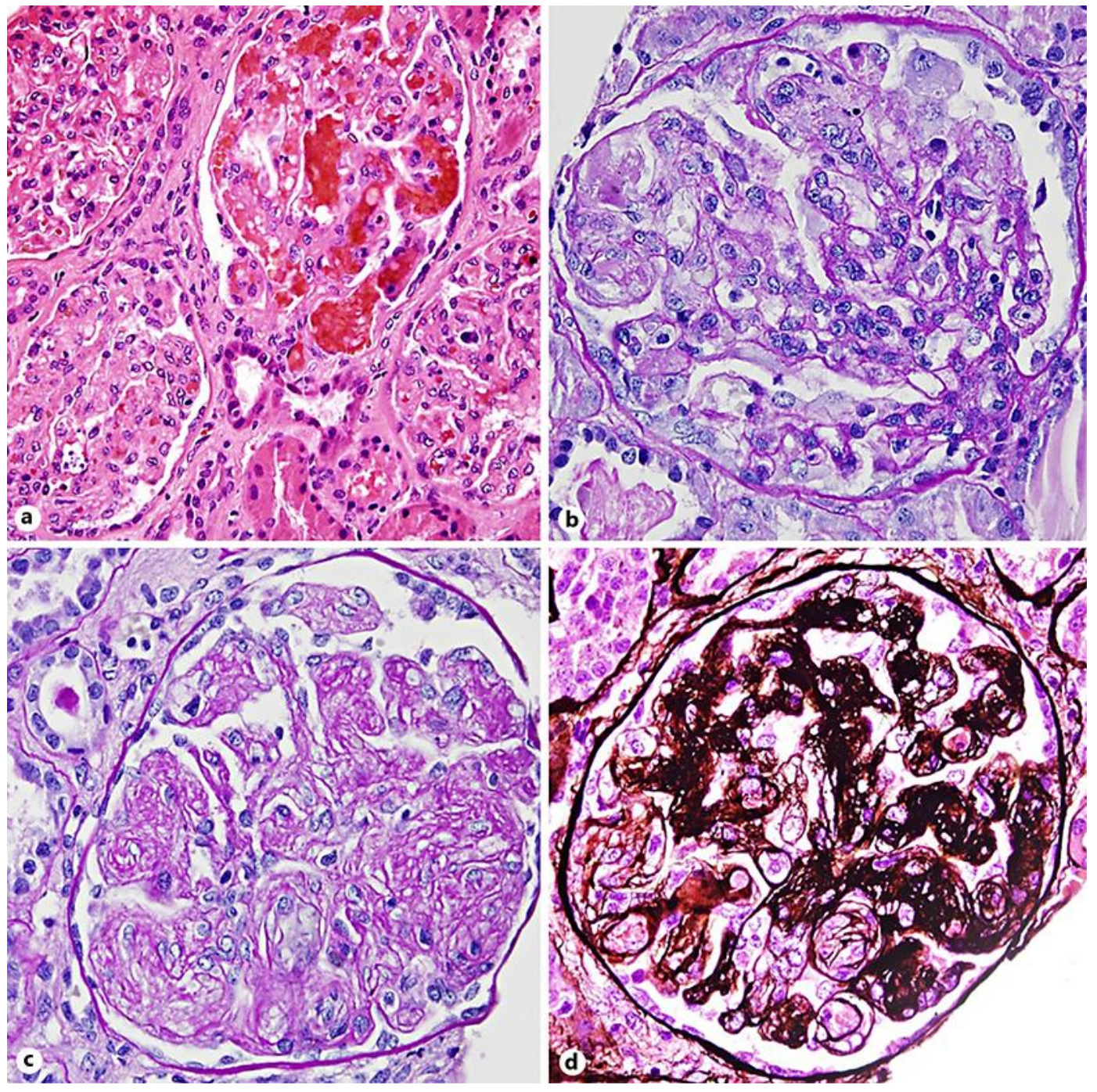

Fig. 1. a Thrombi within glomerular capillary lumina $(\mathrm{HE}, \times 100) . \mathbf{b}-\mathbf{d}$ Endothelial swelling, mesangiolysis, and glomerular basement membrane duplication (b, $\mathbf{c} \times 200$; d PAMS, $\times 200$ ). 